\title{
Inguinal Hernia Repair: Principles and Practice in the United Kingdom
}

\author{
Badri Man Shrestha' \\ 'Department of General Surgery, Sheffield Teaching Hospitals NHS Trust, Sheffield, United Kingdom.
}

It is estimated that approximately 20 million inguinal hernia repair (IHR) are carried out annually worldwide, and IHR is considered as the most frequently performed elective operation in general surgery. IHR is done 10 per 10000 people in the United Kingdom and 28 per 10000 people in the United States. ${ }^{1}$ The surgical techniques used to manage inguinal hernia $(\mathrm{IH})$ are primary open repair (Shouldice and Bassini), open tension-free mesh repair (Lichtenstein repair), and laparoscopic IHR (LIHR) with mesh (Totally extraperitoneal (TEP), Transabdominal preperitoneal (TAPP), Single incision laparoscopic surgery ( SILS) and Robotic repair). IHR can be associated with complications such as surgical site infection (1-5\%), chronic groin pain ( up to $36 \%$ ), ischaemic orchitis and loss of testis (0.2 -1.1\%), injury to vas deferens $(1.6 \%)$, recurrences $(1.1-33 \%)$ and nerve damage (ilioinguinal, iliohypogastric and genitofemoral), which may compromise the quality of life and pose significant financial burden to the patient and the health care provider. ${ }^{2}$

Patients with $\mathrm{IH}$ can present with combination of symptoms and physical signs such as groin pain with or without a clinically palpable lump, an asymptomatic visible and palpable lump, asymptomatic non-visible but a palpable lump, a lump detected by ultrasound scan and a large inguinoscrotal hernia. The natural history of progression of $\mathrm{IH}$ is vague in the literature owing to the traditional approach of operating in almost any patient with the diagnosis of $\mathrm{IH}$. However, in the current climate of constrained hospital resources, setting priority for the management of a wide range of surgical conditions, including cancers, has become paramount. Therefore, best practice guidelines should be formulated for the management of individual presentations of $\mathrm{IH}$, which should be based on the robust evidence, safety of the patient and effective utilisation of available health resources.

In a systematic review of evidence to assess the management of asymptomatic $\mathrm{IH}$, a significant crossover ratio ranging between $23 \%$ and $72 \%$ from watchful waiting to surgery was observed. In patients with watchful waiting, the rates of $\mathrm{IH}$ strangulations were $0.27 \%$ after 2 years of follow-up and $0.55 \%$ after 4 years of follow-up. In patients who underwent elective surgery, the rate of operative complications was $0 \%$ to $22.3 \%$ and the recurrence rate was $2.1 \%$. It was concluded that both options of watchful waiting and surgery are safe, but most patients will develop pain over the time and will require surgery. ${ }^{3}$

In the United Kingdom, hospitals have adopted guidelines for IHR based on the guidelines set by the National Health Service (NHS) of England and National Institute for Health and Care Excellence (NICE), which have proven safe and effective, both for the management of patients and surgical training purposes..$^{4,5}$

For asymptomatic or minimally symptomatic hernias, a watchful waiting approach is advocated where the patient is reassured and clear instruction is given to seek medical attention urgently if features of strangulation develop. Surgical treatment is approved if the hernia becomes symptomatic enough to cause functional impairment, becomes irreducible due to incarceration, progressively increases in size month on month, or presents with an inguinoscrotal hernia. Hernia surgery is not commissioned in patients with groin pain with no visible external swelling. Patients presenting with groin pain who are found to have an impalpable hernia on ultrasound scan should not be referred for hernia repair. Where groin pain is severe and persistent with diagnostic uncertainty, options include referral for musculoskeletal assessment or imaging. Ultrasound should not be routinely requested in the early management of groin pain. ${ }^{4,5}$

Laparoscopic IHR has been introduced with its potential advantages of avoidance of scar, reduced post-operative pain and shorter time to full recovery.

Correspondence: Dr. Badri Man Shrestha, Department of General Surgery, Sheffield Teaching Hospitals NHS Trust, Sheffield, S5 7AU, UK; Email: shresthabm@doctors.net.uk, Fax: +44-1142714604. 
The main drawbacks of LIHR are the need for general anaesthesia, increased cost of the operating room and disposables supplies used and longer learning curve. During LIHR, both TAPP and TEP approaches are utilised at surgeons' discretion; the safety and efficacy both approaches are comparable, except slightly longer hospital stay associated with TAPP. ${ }^{6}$ Both SILS and robotic-assisted LIHR are in their early stages of introduction into routine surgical practice..$^{7,8}$

A meta-analysis including 3297 patients, showed that laparoscopic TEP repair was associated with shorter time to return to work and less chronic pain compared with open Lichtenstein repair. There was no significant difference in incidence of seromas, wound infections and neuralgia. There was significantly higher risk of recurrence after 3 years of LR compared to Lichtestein repair. ${ }^{9}$ For recurrent IH, LIHR showed significantly less wound infection rate and a faster recovery to normal activity, whereas other parameters such as recurrence, pain, reoperation rate, operation time, urinary problems, haematoma and seroma and testicular problems were comparable between two methods. ${ }^{10}$

In the NHS of the United Kingdom, LIHR is not commissioned for primary unilateral hernia repair. Laparoscopic hernia repair is commissioned only for bilateral IHR, where the patient has bilateral IHR with external swelling on clinical examination or for recurrent hernia. LIHR is not commissioned for impalpable hernias found incidentally during laparoscopic repair of a hernia on the other side. Open tension-free mesh repair (Lichtenstein repair) is performed in all patients with unilateral primary $\mathrm{IH}$, and bilateral or recurrent $\mathrm{IH}$ patients, who are unsuitable for general anaesthesia. Majority of IHR are performed as day cases.

The editorial has summarised the basic principles and current practice of IHR in the United Kingdom. However, it is important to adopt a policy of IHR that suits best to the local patient population, which should be guided by available hospital resources and surgical expertise. Regular audit of the main outcome measures such as the duration of surgery, type of anaesthesia, use of antibiotics, length of stay, post-operative complications, analgesic requirements and the costs should be monitored prospectively and appropriate measures taken to make the service effective.

Conflict of Interest: None.

\section{REFERENCES}

1. Ham C. A review of literature. In:Ham C, Ed. Health Care Variations: Assessment in Evidence: Resarch report No. 2. London, England: King's Fund Institute. 1988.

2. Ridgway PF, Shah J, Darzi AW. Male genital tract injuries after contemporary inguinal hernia repair. BJU Int 2002;90(3):272-6

3. Mizrahi H, Parker MC. Management of asymptomatic inguinal hernia: a systematic review of the evidence. Arch Surg 2012;147(3):277-81.

4. NHS England, Interim Clinical Commissioning Policy: Abdominal Wall Hernia Management and repair in Adults. https://wwwenglandnhsuk/commissioning/wp content/ uploads/sites/12/2013/11/N-SC001pdf 2013 (accessed 16 December 2016).

5. Natonal Institute for Health and Care Excellence: Groin hernia guidelines.http://wwwevidencenhsuk/ Search?q=guidelinesthernia 2013 (accessed 16 December 2016)
6. Bracale U, Melillo P, Pignata G, et al. Which is the best laparoscopic approach for inguinal hernia repair: TEP or TAPP? A systematic review of the literature with a network meta analysis. Surg Endosc 2012;26(12):3355-66

7. Far SS, Miraj S. Single-incision laparoscopy surgery: a systematic review. Electron Physician 2016;8(10):3088-95.

8. Cestari A, Galli AC, Sangalli MN, et al. Totally extraperitoneal (TEP) bilateral hernioplasty using the Single Site(R) robotic da Vinci platform (DV-SS TEP): description of the technique and preliminary results. Hernia 2016 Nov 22. [Epub ahead of print] [PMID:27878458]

9. Bobo Z, Nan W, Qin Q, et al. Meta-analysis of randomized controlled trials comparingLichtenstein and totally extraperitoneal laparoscopic hernioplasty in treatment of inguinal hernias. J Surg Res 2014;192(2):409-20.

10. Li J, Ji Z, Li Y. Comparison of laparoscopic versus open procedure in the treatment ofrecurrent inguinal hernia: a meta-analysis of the results. Am J Surg 2014;207(4):602-12. 